Measurements and Phenomenological Modeling of Magnetic FluxBuildup in Spheromak Plasmas

C. A. Romero-Talamas, E. B. Hooper, R. Jayakumar, H. S. McLean, R. D. Wood, J. M. Moller

December 17, 2007

Physics of Plasmas 
This document was prepared as an account of work sponsored by an agency of the United States government. Neither the United States government nor Lawrence Livermore National Security, LLC, nor any of their employees makes any warranty, expressed or implied, or assumes any legal liability or responsibility for the accuracy, completeness, or usefulness of any information, apparatus, product, or process disclosed, or represents that its use would not infringe privately owned rights. Reference herein to any specific commercial product, process, or service by trade name, trademark, manufacturer, or otherwise does not necessarily constitute or imply its endorsement, recommendation, or favoring by the United States government or Lawrence Livermore National Security, LLC. The views and opinions of authors expressed herein do not necessarily state or reflect those of the United States government or Lawrence Livermore National Security, LLC, and shall not be used for advertising or product endorsement purposes. 


\title{
Measurements and phenomenological modeling of magnetic flux buildup in spheromak plasmas
}

\author{
C. A. Romero-Talamás,* E. B. Hooper, R. Jayakumar, \\ H. S. McLean, R. D. Wood, and J. M. Moller \\ Lawrence Livermore National Laboratory, \\ 7000 East Avenue, Livermore, California, 94550. ${ }^{\dagger}$
}

(Dated: December 13, 2007)

\begin{abstract}
Internal magnetic field measurements and high-speed imaging at the Sustained Spheromak Physics Experiment (SSPX) [E. B. Hooper, L. D. Pearlstein, R. H. Bulmer, Nucl. Fusion 39, 863 (1999)] are used to study spheromak formation and field buildup. The measurements are analyzed in the context of a phenomenological model of magnetic helicity based on the topological constraint of minimum helicity in the open flux before reconnecting and linking closed flux. Two stages are analyzed: (i) the initial spheromak formation, i. e. when all flux surfaces are initially open and reconnect to form open and closed flux surfaces, and (ii) the stepwise increase of closed flux when operating the gun on a new mode that can apply a train of high-current pulses to the plasma. In the first stage, large kinks in the open flux surfaces are observed in the highspeed images taken shortly after plasma breakdown, and coincide with large magnetic asymmetries recorded in a fixed insertable magnetic probe that spans the flux conserver radius. Closed flux (in the toroidal average sense) appears shortly after this. This stage is also investigated using resistive magnetohydrodynamic simulations. In the second stage, a time lag in response between open and closed flux surfaces after each current pulse is interpreted as the time for the open flux to build helicity, before transferring it through reconnection to the closed flux. Large asymmetries are seen during these events, which then relax to a slowly decaying spheromak before the next pulse.
\end{abstract}

\footnotetext{
*Electronic address: romerotalamas1@ilnl.gov

${ }^{\dagger}$ The authors are grateful to P. M. Bellan and Caltech for their support to SSPX with the high-speed imaging hardware, to the Center for Magnetic Self-Organization in Laboratory and Astrophysical Plasmas for their financial support, to D. N. Hill, H. Stephens, R. W. Geer, R. O. Kemptner, and M. M. Marchiano for their assistance and guidance during the magnetic probe design, construction and operation. Work performed
} 
under the auspices of the U.S. Department of Energy by Lawrence Livermore National Laboratory in part under Contract W-7405-Eng-48 and in part under Contract DE-AC52-07NA27344. 


\section{INTRODUCTION}

The spheromak is a toroidal confinement concept that contains open and closed magnetic flux surfaces, but is produced in a flux conserver with no coils linking the plasma. The simplicity in its configuration, compared to other confinement concepts, makes spheromaks attractive as fusion reactor candidates. Spheromaks may be produced and sustained in the laboratory using coaxial helicity injection (CHI) [1], among other methods. For reactor relevant spheromaks, the poloidal flux $\Psi(r, z)=\int_{0}^{r} 2 \pi r^{\prime} B_{z}\left(r^{\prime}, z\right) d r^{\prime}$ (where $z$ is the machine axis) must be amplified to factors of a few tens of the initial bias field [2], but the sustaining current must be kept low so as not to damage the CHI source, typically referred to as the spheromak gun. It is therefore of interest to optimize the magnetic flux building process in spheromaks.

At the Sustained Spheromak Physics Experiment (SSPX) [3] research is carried out to investigate how currents in the plasma create and sustain the spheromak, and how fluctuations affect its confinement. In this article, the most recent results are presented of spheromak formation and magnetic flux buildup using CHI, from investigations at SSPX using high-speed imaging and insertable magnetic probes. Focus is given here to a new mode of operating the spheromak gun. Namely, the programming of multiple current pulses throughout a plasma shot.

From this new mode, two distinct stages are considered: the initial plasma ejection and flux buildup during the initial current pulse, and the subsequent current pulses during which both open and closed magnetic flux surfaces might exist. In the first stage current flows only along initially open flux surfaces, which eventually turn into a spheromak with open and closed surfaces through magnetic reconnection. In the second stage, current flows in both open and closed flux surfaces and flux increases stepwise with every gun current pulse until an apparent saturation.

The aim of the present research is to understand the initial spheromak formation process, and the effect of large gun current perturbations on the plasma once the spheromak is formed. Magnetic probes immersed in the plasma and at the flux conserver edge are used to follow the magnetic evolution throughout an entire shot. High-speed images provide up to two images per plasma shot of most of the flux conserver, and complement the magnetic measurements by revealing the formation of large rope-like structures which are well correlated to changes 
in magnetic signals when near the probes.

This article is organized as follows: A phenomenological flux rope model of spheromak formation and flux buildup based on helicity accounting is described in Section II. Magnetic measurements, signal analyses for the formation and buildup stages, and how these measurements relate to the flux rope model are presented in Section III. These analyses include a statistical view at the shot-to-shot signal spread along the insertable probe to infer the level of asymmetry at different times, including the time of the stepwise magnetic signal increase on all the probes, which is conjectured to be caused by a global reconnection event. Comparisons of measurements and two-dimensional numerical reconstruction measurements with a virtual probe are also presented in this section. A brief discussion on numerical simulations of the spheromak formation phase is presented in Section IV.

\section{THE FLUX ROPE MODEL}

The change in spheromak helicity using CHI can be described by the conservation equation $[4]$

$$
\frac{d K}{d t}=2 V_{g} \Psi_{g}-2 \int \eta \mathbf{J} \cdot \mathbf{B} d^{3} r
$$

where $K$ is the total helicity, $V_{g}$ and $\Psi_{g}$ are the gun voltage and gun flux, $\eta$ the plasma resistivity, $\mathbf{J}$ the plasma current, and $\mathbf{B}$ the magnetic field. At SSPX, $V_{g}$ is measured and $\Psi_{g}$ is prescribed by the coaxial gun electromagnets and remains fixed for the duration of an experimental shot since the vessel walls are highly conductive and thus act as a magnetic flux conserver. A typical experimental shot lasts several milliseconds, whereas the flux conserver diffusion time is more than a second. The SSPX gun is designed such that $V_{g} \Psi_{g} \geq 0$, making the total helicity input known for every plasma shot. The second term on the right hand side of Eq. 1, however, represents the losses due to resistive decay and is harder to measure since the plasma currents and magnetic field must be known in the entire volume.

A flux rope model initially proposed to explain the amount of helicity needed for the closing of flux surfaces shortly after plasma breakdown in SSPX [5], is extended here to include the stepwise flux buildup seen during runs with multi-pulse gun current waveforms. The model, which is based on the idea originally presented by Bellan [27] about the magnetic field acquiring a minimum of two turns of helicity before changing topology, is shown schematically in Fig. 1. The bias field has zero helicity, and is represented by a straight 
rope in Fig. 1(a). Immediately after plasma breakdown, current flows along the bias flux surfaces and helicity starts to increase until it achieves the minimum amount necessary to create closed surfaces through magnetic reconnection. In the flux rope model this condition is equivalent to two full turns in the rope, as shown in Fig. 1(b). The addition of helicity in the closed flux surfaces can be quantized to multiples of two full turns provided helicity is unchanged during reconnection, as shown in Fig. 1(c), where the two rings are assumed to eventually merge and form a single ring.

\section{A. Equivalence between twists and linkage in a flux rope}

For the reader unfamiliar with concepts of helicity, the condition of two full turns in a flux rope before linkage might seem arbitrary. However, the magnetic helicity equivalence between linked ropes and twisted or writhed ones is well known and has been described by several authors. Bellan, for example, presents a compilation of previous references and a concise derivation of helicity accounting for linked and twisted ropes in Ref. [6]. These derivations are summarized here for completeness.

We show below how magnetic helicity in a volume $V$, defined by

$$
K=\int_{V} \mathbf{A} \cdot \mathbf{B} d^{3} r
$$

where $\mathbf{A}$ is the vector potential and $\mathbf{B}=\nabla \times \mathbf{A}$ the magnetic field, is the same for both cases in Fig. 1(b). A may be divided in toroidal and poloidal components (with $\phi$ and $\theta$ being their respective angles)

$$
\mathbf{A}=\mathbf{A}_{p o l}+\mathbf{A}_{t o r}
$$

which are related to the toroidal flux, $\phi$, and the poloidal flux, $\Psi$ by

$$
\begin{aligned}
\mathbf{A}_{\text {pol }} & =\frac{\Phi}{2 \pi} \nabla \theta \\
\mathbf{A}_{\text {tor }} & =\frac{\Psi}{2 \pi} \nabla \phi
\end{aligned}
$$

so that

$$
\mathbf{B}=\frac{1}{2 \pi}(\nabla \Phi \times \nabla \theta+\nabla \Psi \times \nabla \phi) .
$$

Substituting these into Eq. 2, one finds [6]

$$
K=[\Phi \Psi]_{0}^{\Psi_{\max }}-2 \int_{0}^{\Psi_{\max }} \Phi d \Psi
$$


Setting $\Psi=0$ at the wall of a flux rope inside $V$ implies $\Psi_{\max }$ occurs at the magnetic axis. Conversely, the magnetic axis has $\Phi=0$, and $\Phi_{\max }$ occurs at the rope wall. With this convention the $\Psi \Phi$ term in Eq. 7 evaluates to zero, and changing the limits of integration to be functions of $\Phi$ reduces the equation to

$$
K=2 \int_{0}^{\Phi_{\max }} \frac{d \Psi}{d \Phi} \Phi d \Phi .
$$

Note that by definition of a flux rope, the flux is constant along the rope, and thus $\Phi$ will be used to describe the total flux along the rope hereafter.

Let $T=d \Psi / d \Phi$, which is the rotational transform divided by $2 \pi$, be the turns in the poloidal direction per turns in the toroidal direction. Then, if $T$ is constant,

$$
K=T \Phi^{2}
$$

This is the expression that allows us to account for helicity in an isolated rope with flux $\Phi$ and $T$ turns.

We now wish to find the total helicity in a volume containing two linked, untwisted flux ropes. Each rope occupies a volume $V_{i}$, has a flux $\Phi_{i}$, and a helicity $K_{i}(i=1,2)$. Eq. 2 is used for each rope, and the total helicity in the volume enclosing both ropes is simply

$$
K=K_{1}+K_{2}
$$

To evaluate $K_{1}$ we note that $d r^{3}=d \mathbf{l} \cdot d \mathbf{S}$, where both $d \mathbf{l}$ and $d \mathbf{S}$ are parallel to the rope, then

$$
K_{1}=\oint_{C_{1}} \int_{S_{1}}(\mathbf{A} \cdot d \mathbf{l})(\mathbf{B} \cdot d \mathbf{S})
$$

where $C_{1}$ is the contour along the flux tube and $S_{1}$ its cross-section, $\Phi_{1}=\int_{S_{1}} \mathbf{B} \cdot d \mathbf{s}$ is constant along the flux tube, and thus

$$
K_{1}=\Phi_{1} \oint_{C_{1}} \mathbf{A} \cdot d \mathbf{l} .
$$

Noting that magnetic flux is zero outside any of the flux ropes, and rope 2 links rope 1,

$$
\oint_{C_{1}} \mathbf{A} \cdot d \mathbf{l}=\Phi_{2}
$$

and thus $K_{1}=\Phi_{1} \Phi_{2}$. Repeating the steps for rope 2 gives $K_{2}=K_{1}$, and therefore

$$
K=2 \Phi_{1} \Phi_{2}
$$


Clearly, if $\Phi_{1}=\Phi_{2}=\Phi$ in Eq. 14 and $T=2$ in Eq. 9, then the helicity (but not the topology) is the same for both cases. The additive property of helicity allows us to extend the above results to account the helicity in Fig. 1(c), $K=4 \Phi^{2}$, and more generally

$$
K_{n}=2 n \Phi^{2}
$$

where $n=1,2,3, \ldots$ is the number of stacked rings (provided, of course, helicity does not decay during the stacking process).

It should be pointed out that for a fixed volume in which the stacking occurs helicity may be conserved, but after the stacked rings merge, the toroidal flux in the final ring need not be the algebraic sum of the flux in the stacked rings [6].

\section{B. Helicity buildup and saturation}

For a linked plasma rope bounded by a flux conserver, the addition of new rings becomes increasingly difficult with every new ring for a limited power source, i.e., flux in the closed surfaces saturates. Since the flux rope model relies on the central column kinking, closed flux saturation can be explained in terms of the Kurskal-Shafranov stability condition for a cylindrical column with an axial magnetic field $q(a)=(2 \pi a)^{2} B_{z} L \mu_{o} I>1$, where $a$ is the column radius, $L$ the column length, and $I$ the current through the column [7]. The column begins to kink at $q(a)=1$, giving the critical current

$$
I_{c}=\frac{(2 \pi a)^{2} B_{z}}{L \mu_{o}} .
$$

In SSPX the column length is approximately fixed at $L=0.5 \mathrm{~m}$, yielding $I_{c} \rightarrow I_{c}\left(a, B_{z}\right)$. This threshold might be lower in SSPX since one of the column ends is not line tied and

there is finite resistivity at the CHI electrodes [8]. However, this level of detail is omitted here in favor of the phenomenological insight that Eq. 16 provides.

In the context of the flux rope model, $B_{z}$ increases at the addition of every ring since toroidal currents in these rings are assumed to be added faster than they can decay between reconnection events. On the other hand, plasma temperature cannot increase significantly along open channels with constant $L$. The column radius magnitude thus results from a competition between the pressure from the closed flux surfaces and the magnetic pressure from the $B$ field in the open flux surfaces. The plasma thermal to magnetic pressure ratio 
$\beta_{e} \equiv 2 n k T_{e} / \mu_{o} B^{2}$ is typically of a few percent in the closed region, and much smaller in the open region.

The concept of helicity saturation for a train of gun current pulses in SSPX was discussed by Woodruff et al. [9], who derived a limiting helicity $K_{\infty}=\Delta K_{g}\left[1-\exp \left(-\Delta t / \tau_{K}\right)\right]^{-1}$ from Eq. 1 , by assuming a constant helicity pulse $\Delta K_{g}$ with a duration $\Delta t$ and a fixed helicity decay $\tau_{K}$. They conjectured that if $\tau_{K} \rightarrow \infty$ (i. e., resistive decay becomes negligible), then helicity buildup would not saturate. However, a constant $\Delta K_{g}$ implies a constant $V_{g}$ profile at each pulse, and as it will be shown in Sec. III B, $V_{g}$ actually decreases (on average) at every pulse. This may be interpreted in terms of the flux rope model and Eq. 16 as the central column becoming increasingly stabilized from the addition of closed flux, which in turn decreases the possibility of inductance changes in the column that would then contribute to $V_{g}$.

It should be noted that the present model only requires a minimum of helicity before every reconnection event, but not a maximum. An open rope could exceed the required helicity before reconnecting, and transfer part or all of that excess helicity to the closed flux. Therefore, geometrically distorted structures (i.e. non-symmetric after reconnection) are contemplated within this model. There are also no restrictions about the rotation of open or closed structures. Unwinding of the open flux surfaces would remove helicity and would represent the resistive decay of Eq. 1 for these surfaces. Unwinding closed flux, on the other hand, would not be as simple since it would require disconnection of the excess closed flux and merging or reconnection of this flux to the open one. Flux surfaces could then become fully open for the duration of the closed surfaces unwinding.

Boozer [10] showed that closed flux surfaces with time and volume averaged parallel currents cannot be sustained continuously by a dynamo. That is, closed surfaces must be opened, at least intermittently, to maintain or increase helicity in them. Moses et al. [11] then showed explicitly that this imposes a constraint on plasma temperature due to enhanced transport. This is certainly consistent with the model presented here, noting that the sequence shown in Fig. 1(c) increases the amount of closed flux in a two step process: each additional ring first disconnects from the open flux rope and then merges with the existing closed flux. Here closed flux need not connect with the open flux surfaces unless the time between the two reconnection steps becomes zero.

This apparently contradicts Moses et al. assertion that helicity addition entails direct 
connection to the open flux during helicity addition and consequently some loss of closed flux energy. Their work, however, assumed the closed flux to be simply connected, and the two rings in Fig. 1(c) are not, at least before the two rings merge (which opens flux surfaces, but only within the closed flux), and thus there is no contradiction.

\section{EXPERIMENTS}

Experimental data from SSPX is now analyzed from the perspective of the flux rope model. The model of course focuses on topological restrictions of the helicity needed to build and maintain linked flux surfaces, and is not intended to represent every detail of the spheromak experiments. Instead, phenomenological elements of the model are compared to experimental evidence.

For the experiments presented here, a specially adapted high-speed imaging system [12] and an insertable magnetic probe (similar in construction to that described in Ref. [13]) with a total of 20 clusters of chip inductors spaced every $2 \mathrm{~cm}$, were used to follow plasma evolution and measure the three components of the magnetic field along the flux conserver radius $(50 \mathrm{~cm})$. The camera was used to take up to two images per plasma shot (visible light, mostly $H_{\alpha}$ ) with a horizontal field of view of almost $1 \mathrm{~m}$ and a vertical field of view of more than $0.5 \mathrm{~m}$ (the height of the flux conserver). The probe was fixed for the duration of a plasma shot, covered in a boron nitride sheath to protect it from arcing or melting, and to provide electrical isolation from the plasma.

Edge magnetic signals with and without the probe inserted showed the signals build to lower amplitudes and decay faster with the probe inserted, due most likely to the increased resistivity (lower plasma temperature). Qualitatively, however, the signals were the same for both cases. Plasma density was measured using a $\mathrm{CO}_{2}$ laser interferometer [14], and was relatively flat with values $(0.5-1) \times 10^{20} \mathrm{~m}^{-3}$ for the shots discussed in Section III B. These values are typical for spheromak shots with or without the probe inserted. For the formation only shots (Section III A), the density rises to a factor of a few above $1 \times 10^{20} \mathrm{~m}^{-3}$ for tens of microseconds. Here too, plasma density with the probe inserted was found to be qualitatively similar to the case without the probe. 


\section{A. Initial plasma ejection from the gun and spheromak formation}

Initially only a pre-programed bias magnetic field exists inside the flux conserver. The coils that produce this field are turned on seconds before plasma breakdown in order for the field to diffuse through the flux conserver and reach equilibrium, resulting in the gun flux being fixed and known for the duration of a given plasma shot, as shown in Fig. 2. A neutral gas (typically hydrogen) is injected through gas-puff valves, and a short time later, a potential of a few kilovolts is applied between the electrodes. Plasma then breaks down $(t=0)$ and current begins to flow between the electrodes. This process had been described previously in Refs. [5, 12] from findings using only high-speed images and edge magnetics. With these and other diagnostics, it has been found that plasma may enter the flux conserver region symmetrically or asymmetrically.

The symmetric expansion case is shown in Fig. 3. Magnetic probe measurements indicate that during the initial plasma expansion, currents flow along a current sheet that is less than $10 \mathrm{~cm}$ wide, and which essentially makes the boundary of the expanding bubble. The toroidal bubble eventually fills the flux conserver and coalesces to form the central column seen in Fig. 3. The magnetic field's poloidal and toroidal components indicate that the field inside the column is purely poloidal, and outside of it purely toroidal. Nevertheless, the central column is not static. It continuously evolves as the gun current keeps increasing at the level of hundreds of kilo-amperes, eventually developing a large kink which coincides with a large gun voltage spike that is conjectured to precede magnetic reconnection which in turn leads to closed magnetic flux surfaces. Examples of columns kinking during this time are shown in Fig. 4.

The initial column kink would correspond to Fig. 1(a) in the flux rope model. However, resistivity and helicity decay are expected to be significant during this time and compete with the helicity input from the gun. Closed flux rapidly disappears or not appears at all. Nevertheless, the gun current is uninterrupted during this time, so the process repeats itself until resistivity in the closed flux is sufficiently low for plasma currents to sustain it and stabilize the open column.

Typical gun current and voltage traces $\left(I_{g}\right.$ and $\left.V_{g}\right)$ for formation-only shots are shown in Fig. 5. The inset images, taken shortly after plasma first appeared in the flux conserver, represent an example of asymmetric breakdown. In this case, plasma first appeared at 
approximately 45 degrees of the flux conserver reference (left side of the image field of view) and by $t=55 \mu \mathrm{s}$ the plasma had rotated towards 90 degrees. The bright feature seen at the center of the right image is caused by a large current intercepting the probe tip. Structure is also observed within the ejected plasma, probably caused by filamentation in the gun region $[15]$.

Whether plasma is ejected symmetrically or not, or the level of ejection asymmetry, are at present not controllable parameters in SSPX. These probably depend on variables such as the initial breakdown location, wall recycling, etc. However, magnetic self-organization leads to periods of high reproducibility, depending on the gun current and magnetic flux levels. This can be sen in Figure 6(e), which shows small uncertainty bars on the average of 16 shots with the same current profile shown in Fig. 5. Note that these bars represent the shot-to-shot spread in the magnetic field at any given time, and exclude the systematic errors from hardware or software processing. These systematic errors do not affect the observations and discussions presented hereafter.

The pulse forming network acts as a current source in SSPX [9], making $I_{g}$ a controllable and reproducible parameter (better than 98\%) depending mainly on the charge voltage of the capacitor bank. The gun voltage on the other hand, results mainly from the resistive and inductive components of the plasma which obviously vary at a much higher frequency than the gun current. It is then remarkable that many features of the gun voltage are also highly reproducible, and in particular the first high-voltage spike, which occurs at $70 \pm 5 \mu s$ for this particular set of shots. By this time, plasma becomes highly ionized and thus difficult to capture with high-speed images. As mentioned above, images captured shortly before the voltage spike, indicate the development of large kinks such as those shown in Fig. 4. These images support the estimates presented in Ref. [9] that at this time $V_{g} \simeq I_{g} d L / d t$ (where $L$ is the plasma inductance).

The spread bars in the toroidal signals in Fig. 6(a) show the uncertainties in where the current flows and where the kink starts (with respect to the probe). The poloidal component, however, have significant spread bars up to $0.2 \mathrm{~m}$ and from $0.4 \mathrm{~m}$ to the wall, but very small signal and uncertainties in-between. Edge magnetic probes indicate a structure that rotates inside the flux conserver at this time, consistent with the formation of an $n=1$ asymmetry with the so-called "dough-hook" shape $[16,17]$.

As time progresses, the gun current keeps increasing and so does the field magnitude in 
both the poloidal and toroidal components, but not their respective uncertainties. At $100 \mu s$ [Fig. 6(b)] the toroidal error bars decrease close to the machine axis, but the poloidal ones increase significantly. This is indicative of the dough-hook asymmetry still present, but without reaching the flux conserver edge at the probe level, since the poloidal uncertainty is small near the region. The poloidal field field magnitude throughout the probe is also indicative of toroidal currents which by now might be in closed surfaces (at least in the toroidally averaged sense), or have a long connection length.

The highest uncertainty levels in both the toroidal and poloidal directions is seen at $110 \mu s$ [Fig. 6(c)]. Notice, however, that the toroidal uncertainties have a minimum at $R=26 \mathrm{~cm}$. This is conjectured here to be caused by a prominent rotating kink in the open flux, and the perturbation this kink creates on the toroidal current linking the open flux. The second highest voltage spike (Fig. 5) characteristic in all the shots also appears at this time. This time is of great interest to understand flux buildup, and is discussed further in the sections below.

At $120 \mu s$ [Fig. 6(d)] the uncertainties closer to the machine axis decrease, particularly in the poloidal field, but now those between radii 0.3 and $0.4 \mathrm{~m}$ increase. Conversely, the toroidal uncertainties decrease in this range. By $t=150 \mu \mathrm{s}$ uncertainties decrease to small values, and at $190 \mu s$ [Fig. 6(e)] they reach their smallest values (except for the toroidal signals near the edge, which oscillate around zero). This indicates the most stable time for the driven spheromak, since any rotating distortion on the magnetic flux surfaces would tend to increase the spread bars.

With uncertainties still relatively small, the maximum field amplitude is achieved at $210 \mu s$ [Fig. 6(f)]. Note that $V_{g}$ in Fig. 5 is close to zero at this time. This maximum lasts no longer than $10 \mu s$, and gradually decreases thereafter. The last $V_{g}$ small spike $(\sim 100 \mathrm{~V})$ coincides with a brief increase in uncertainties, shown in Fig. 6(i). As the magnetic field gradually decreases, the uncertainties remain relatively low for the rest of the shot.

\section{B. Multiple current pulses}

A recent modification to the SSPX power system enables independently triggered capacitors to deliver up to $50 \mathrm{kA}$ each at different times during a plasma shot [18]. Pulsed gun current waveforms were programmed to investigate spheromak evolution during consecutive 
pulses. A typical shot from this set of experiments is shown in Fig. 7(a). The gun voltage varied at every pulse [Fig. 7(b)] as a result of resistive and inductive changes in the plasma. The typical $|B|$ signals for these shots are shown in Fig. 7(c), with the higher amplitude signals closer to the geometric axis, and the lower amplitude ones closer to the flux conserver edge.

Note that in these shots, $|B|$ increases stepwise with every current pulse until reaching an apparent saturation by the fourth pulse. However, the most remarkable feature in these signals is the difference in response time at different probe locations. The probes located where open flux surfaces are expected (intercepting the electrodes, as shown in the left side of Fig. 2) have an essentially immediate response to each current pulse. In contrast, the probes immersed where closed surfaces are expected have a time lag of more than $120 \mu s$ before the field stops decaying and starts to rise (i.e., $d B / d t$ changes sign over a few microseconds). The time lag at each pulse is indicated with a pair of dotted lines in Fig. 7, and in the close-up of the fourth pulse in Fig. 8.

The closed flux response marks an inflection point for all the signals, that is, a global magnetic event takes place in a time-scale of a few microseconds, followed by an increase in magnetic energy in the closed flux signals and a momentary flattening or decrease in magnetic energy in the open ones. Based on this evidence it may be argued that at this time magnetic reconnection, and not simply resistive diffusion, transfers magnetic flux from the open flux surfaces to the closed ones. In the flux rope model, this stage corresponds to Fig. 1(c).

In order to compare time scales, dimensional analysis on a cylindrical conducting shell [19] may be used to estimate the resistive diffusion time for the toroidal closed region,

$$
\tau_{R}=\frac{\mu_{o} r \delta}{2 \eta},
$$

where $r$ is the cylinder radius, $\delta$ the wall thickness, and $\eta$ the plasma resistivity. SSPX plasmas with probes inserted are relatively cold and have $T_{e} \sim 10-20 \mathrm{eV}$ (as measured from a different set of shots). Using Spitzer resistivity [20] with an effective charge state $Z_{\text {eff }} \sim 2$, a Coulomb logarithm $\ln \Lambda \sim 15$, and $T \sim 10 \mathrm{eV}$, then $\eta \sim 9.8 \times 10^{-5} \Omega \mathrm{m}$. For a solid conductor cylinder $\delta=r$. From the number of traces measuring the open flux in Fig. $8, r \sim 0.18 \mathrm{~m}$ (since probes are $2 \mathrm{~cm}$ apart, and assuming that the closed surfaces reach near the flux conserver wall, which is $0.5 \mathrm{~m}$ away from the geometric axis). Evaluating Eq. 
17 with the above estimates yields $\tau_{R} \sim 200 \mu s$.

This time is of course comparable to that shown in Fig. 8. However, this is an estimate of the response time for the channels close to the magnetic axis $(r=0$, approximately channel 16 in the figures). Resistive diffusion would have yielded a gradual response in the signals between the open region and the magnetic axis, which is not observed. Only the signal at the open flux boundary (channel seven) exhibits a delayed response of about $50 \mu s$. The above $\tau_{R}$ estimate is therefore likely to be low for the closed flux region, and plasma temperatures higher than $20 \mathrm{eV}$ should be used.

The last pulse in Fig. 7(c) shows a different behavior between the dotted lines from the previous two pulses. By this time, flux buildup has saturated. The helicity injection rate from the plasma gun also decreases for the last two pulses, as shown in Fig. 9. Note that the pulse amplitude and duration is not identical for all the pulses, with the duration of the first two pulses being less than the rest. As mentioned above, flux buildup saturation is understood in terms of the flux rope model as the consequence of the stabilization provided by the added helicity in the closed flux, and the increased difficulty for the column to change its inductance.

The conjectured reconnection event is also seen at the last dotted line, but before that occurs the field in the closed flux region does respond to the gun current increase. This is probably caused not by field diffusion effects considered above, but by large asymmetries that perturb simultaneously the open and closed flux regions until reconnection finally occurs.

The exercise of looking at the shot-to-shot spread for multiple current pulse shots has been carried out for the same waveform as the one shown in Fig. 7. The results are shown in Fig. 10. Note that for these experiments the probe was placed closer to the machine axis than for the cases shown in Fig. 6 .

Just before the fourth pulse starts, Fig. 10(a) shows the spread in both toroidal and poloidal signals closer to the geometric axis, indicative of symmetry breaking modes in the open flux surfaces. As the current is ramped up these modes tend to disappear around $\lambda_{g}=10.5 \mathrm{~m}^{-1}$ [Fig. 10(b)], and then rapidly grow again when approaching $\lambda_{g}=12 \mathrm{~m}^{-1}$ [Fig. 10(c)]. This is the onset of the conjectured reconnection event, indicated in the second dashed line in Fig. 8. The shot-to-shot spread bars continue to grow along the entire probe for more than $100 \mu s$ [Figs. 10 (d), (e), and (f)], as $I_{g}$ passes through the pulse maximum. The spread eventually decreases [Fig. 10(g)], but the average values for the magnetic field 
continue to increase [Fig. 10(h)] well after the pulse maximum, followed by a slow decrease with small spread along the probe until the next $I_{g}$ pulse starts.

\section{Equilibrium reconstructions}

Measurements of $|B|$ along the probe have also been compared to reconstructions from the two-dimensional Grad-Shafranov solver code CORSICA [21] at different times during multi-pulse shots. The typical output for a CORSICA reconstruction is shown at the left of Fig. 2. These reconstructions use edge magnetic measurements as boundary conditions to constraint the calculations, but not the internal measurements provided by the probe. The insertable probe thus provided independent internal measurements to compare against CORSICA reconstructions. The comparison between SSPX measurements and a virtual probe in CORSICA are shown in Fig. 11 for the decay phase of the third pulse shown in Fig. 7.

It should be noted that CORSICA did not yield reconstructions for all times during the shots (i.e., the program did not converge to a physical solution), and in particular for those times with large field asymmetries. Edge magnetic measurements for shots that resulted in reconstructions like the one shown in Fig. 2, as well as profiles for which CORSICA reconstructions were unacceptable, are shown in Fig. 12. The measurements shortly after the conjectured reconnection event (solid line) did not yield an equilibrium reconstruction, while the measurements taken as the spheromak is slowly decaying (dashed line) yield the aforementioned reconstructions.

In terms of the flux rope model, the magnetic edge profile that results shortly after reconnection is expected to be higher than in the relaxed case close to the gun $\left(B_{p}\right.$ coil 1 in Fig. 12), since the news ring would carry currents close to that region and would not merge immediately with the preexisting closed flux. After the merging the field close to the gun decreases, but the field closest to the flux conserver axis $\left(B_{p}\right.$ coil 17$)$ increases since now more current has been added in the toroidal direction in that region.

Using CORSICA reconstructions of magnetic flux in the open surfaces, it is possible to obtain a semi-quantitative estimate of the time required to buildup enough helicity before reconnection, and compare the helicity level during this time to the flux rope model. For the fourth pulse in Fig. 7., this comparison is shown in Fig. 13. The horizontal lines 
represent the helicity for different number of rings, $n$ according to Eq. 15, where the flux $\Phi=(17.8 \pm 0.182) \times 10^{-3} W b$ is the average and standard deviation of flux reconstructed from shots 16208 to 16210 at the start of the pulse, represented by the first dotted line. Note that this standard deviation does not take into account reconstruction errors. The second dotted line is the start of the conjectured reconnection event, and the third dotted line marks the time at which helicity approximately reaches the next ring level in the flux rope model. Interestingly, the figure shows the seemingly stepwise helicity buildup up to approximately $t=1.8 \mathrm{~ms}$, reaching a maximum at $n=6$ for about $100 \mu \mathrm{s}$, and then gradually decaying after that.

The absolute errors for the open flux and total helicity calculations in CORSICA are not known, as there is no independent measurement for these quantities in the experiment. Conservative error estimates have been performed by comparing CORSICA output with respect to the root mean square error in the edge magnetic field. These estimates yielded a relative uncertainty in helicity magnitude of $\pm 20 \%$, and of $\pm 10 \%$ in flux through the center column. Note that these uncertainties are not plotted in Fig. 13.

The CORSICA uncertainties are of course too big to ascertain agreement between the $2 n \Phi^{2}$ levels and the reconstructions. Furthermore, shortly after the pulse starts, the flux along the column actually increases at a faster rate than helicity in the entire volume, and this change is not reflected over time in the $n$ levels shown in the figure. This indicates that the helicity decay rate in the closed flux is probably higher during this time than later in the pulse. With these uncertainties, the best time estimate for the addition of a new ring in the flux rope model is at $t=1.7 \mathrm{~ms}$ in Fig. 13, which is within a factor of about 1.5 with respect to the time at which reconnection is conjectured to start (second dotted line).

\section{NUMERICAL SIMULATIONS}

Numerical simulations of early spheromak formation in SSPX using a three-dimensional resistive magnetohydrodynamic code, NIMROD [22], are discussed here. Although the code does not include plasma breakdown or atomic physics, it closely reproduces the initial plasma ejection from the gun discussed in Section III A, including the hollow current profile measured in the plasma column [23].

A series of current density plots is shown in Fig. 14 for this initial stage. However, 
the appearance of current structures inside the toroidal "bubble" is not observed in the experiments during the ejection stage. These toroidal currents in the simulation create poloidal flux inside the bubble even before reaching the flux conserver bottom. Toroidal modes numbers with $n=1$ and higher develop shortly after the bubble has reached the flux conserver bottom, but the central column displacement is not as large as that seen in the experiments. Nevertheless, the first sign of reconnection in the simulations coincide with the first large voltage increase [24].

Closer inspection on the toroidal currents inside the bubble reveal that these currents, which appear as soon as $t=38.8 \mu \mathrm{s}$ in Fig. 14, are not actually connecting or "shorting" the bubble current path, but are likely created by induction from the combination of the rapid bubble expansion (and thus a rapid inductance change) and the steep current increase. The appearance of induced currents is also facilitated by the initial background plasma, which is necessary for simulations to converge. This contrasts with the experimental initial condition, where the bubble expands into an initially vacuum flux conserver, and where plasma is possibly only present at the bubble surface (current sheet) and not inside it. The latter has not been verified experimentally since density measurements are line integrated and cannot resolve the density profile inside the expanding bubble.

Investigations of multiple gun current pulses with NIMROD are ongoing and will be reported elsewhere.

\section{CONCLUSION}

The experimental measurements of spheromak formation and flux buildup presented here provide strong indications of repeated and reproducible magnetic reconnection that changes the global topology and increases the magnetic flux in a stepwise fashion. The time lag between the start of each pulse and the increase in magnetic field where the closed flux surfaces are expected, is interpreted as the time to build enough helicity in the open surfaces before reconnection transfers flux to the closed ones. The shot-to-shot statistical spread in the probe signals indicate large amplitude asymmetries that develop during the conjectured reconnection process, with the low spread appearing during the relaxation stage of each current pulse.

This evidence is consistent with the flux rope model described in this article. To date, 
however, the model is only phenomenological and not quantitative, due to a lack of measurements and precision that would allow the estimation of the total helicity, the helicity decay rate, and the reconnection details at the open and closed flux surfaces. Better understanding of these parameters would be required for spheromak reactor scenarios in which poloidal flux is amplified and sustained by periodically pulsing the gun current and controlling the flux conserver response (such as the so-called "intelligent" wall, described by Bishop [25] and recently tested in a reversed-field pinch by Brunsell et al. [26]).

[1] T. R. Jarboe. Plasma Phys. Control. Fusion, 36:945, 1994.

[2] R. L. Hagenson and R. A. Krakowski. Fusion Technol., 8:1608, 1985.

[3] E. B. Hooper, L. D. Pearlstein, and R. H. Bulmer. Nucl. Fusion, 39:863, 1999.

[4] B. W. Stallard, Hooper, S. Woodruff, R. H. Bulmer, D. N. Hill, H. S. McLean, and R.D. Wood. Phys. Plasmas, 10(7):2912, 2003.

[5] C. A. Romero-Talamás, C. Holcomb, P. M. Bellan, and D. N. Hill. Phys. Plasmas, 13(12):022502, 2006.

[6] P. M. Bellan. Spheromaks: A Practical Application of Magnetohydrodynamic Dynamos and Plasma Self-Organization, volume 12. Imperial College Press, 2000. Chapter 3.

[7] G. Bateman. MHD Instabilities. The MIT Press, Cambridge, Massachusetts, 1978. Chapter 6.

[8] D. D. Ryutov, I. Furno, T. P. Intrator, S. Abbate, and T. Madziwa-Nussinov. Phys. Plasmas, 13(3):032105, 2006.

[9] S. Woodruff, B. I. Cohen, E. B. Hooper, H. S. McLean, B. W. Stallard, D. N. Hill, C. T. Holcomb, C. Romero-Talamas, R. D. Wood, G. Cone, and C. R. Sovinec. Phys. Plasmas, 12:052502, 2005 .

[10] A. H. Boozer. Phys. Fluids B, 5:2271, 2006.

[11] R. W. Moses, R. A. Gerwin, and K. F. Schoenberg. Phys. Plasmas, 8:4839, 2001.

[12] C. A. Romero-Talamás. Investigations of spheromak plasma dynamics: high-speed imaging at the sustained spheromak physics experiment and magnetic diagnostics at the Caltech spheromak experiment. PhD thesis, California Institute of Technology, Pasadena, California, 2005.

[13] C. A. Romero-Talamás, P. M. Bellan, and S. C. Hsu. Rev. Sci. Instrum., 75:2664, 2004. 
[14] H. S. McLean, A. Ahmed, D. Buchenauer, et al. Rev. Sci. Instrum., 72:556, 2001.

[15] D. D. Ryutov, R.H. Cohen, and D. N. Hill. Plasma Phys. Rep., 29(7):605, 2003.

[16] R. C. Duck, P. K. Browning, G. Cunningham, S. J. Gee, A. al Karkhy, R. Martin, and M. G. Rusbridge. Plasma Phys. Control. Fusion, 39:715, 1997.

[17] C. R. Sovinec, J. M. Finn, and D. del Castillo-Negrete. Phys. Plasmas, 8:475, 2001.

[18] M. M. Marchiano, E. G. Cook, R. W. Geer, et al. 2005. Proceedings $15^{\text {th }}$ IEEE International Pulsed Power Conference, Monterey, CA. 0-7803-9198-6/05.

[19] R. H. Loveberg. Plasma Diagnostic Techiques. edited by R. H. Huddleston and S. L. Leonard, Academic Press, New York, 1965. Chapter 3.

[20] L. Spitzer. Physics of Fully Ionized Gases. Interscience, New York, 1962.

[21] A. Tarditi, R. H. Cohen, G. C. Craddock, J. A. Crotinger, G. D. Porter, T. D. Rognlien, A. I. Shestakov, and G. R. Smith. Contrib. Plasma Phys., 36:132, 1996.

[22] C. R. Sovinec, A. H. Glasser, T. A. Gianakon, D. C. Barnes, R. A. Nebel, S. E. Kruger, D. D. Schnack, S. J. Plimpton, A. Tarditi, and M. S. Chu. J. Comp. Phys., 195:355, 2004.

[23] C. A. Romero-Talamás, E. B. Hooper, D. N. Hill, B. I. Cohen, H. S. McLean, R. D. Wood, and J. M. Moller. J. Fusion Energy, 26:169, 2007.

[24] E. B. Hooper, T. A. Kopriva, B. I. Cohen, D. N. Hill, H. S. McLean, R. D. Wood, S. Woodruff, and C. R. Sovinec. Phys. Plasmas, 12(9):092503, 2005.

[25] C. M. Bishop. Plasma Phys. Control. Fusion, 31:1179, 1989.

[26] P. R. Brusnell, M. Kuldkepp, S. Menmuir, M. Cecconello, A. Hedqvist, D. Yadikin, J. R. Drake, and E. Rachiew. Nucl. Fusion, 46:904, 2006.

[27] See EPAPS Document No. E-PHPAEN-12-034512 for P.M. Bellan's Innovative Confinement Workshop oral presentation (Madison, 2004). A direct link to this document may be found in the online articles HTML reference section. The document may also be reached via the EPAPS homepage (http://www.aip.org/pubservs/epaps.html) or from ftp.aip.org in the directory /epaps. See the EPAPS homepage for more information. 


\section{FIGURE CAPTIONS}

- Figure 1. Helicity addition and topology changes. For an individual rope, helicity $K$ is the number of times the dark stripe goes around the rope between its ends: (a) $K=0 ;($ b) $K=2 ;$ (c) $K=4$.

- Figure 2. (Color) Typical vacuum flux contours (right) and CORSICA reconstruction of poloidal flux during a shot (left) [adapted from Ref. [9] with kind permission from the author]. The insertable magnetic probe array is shown right. The tick marks along the probe indicate the location of each cluster which are used to measure Bx, By, and $\mathrm{Bz}$, and correspond to the traces shown in Fig. 7. Cluster 1 is closest to the geometric axis, and cluster 20 is the closest to the flux conserver wall. Edge magnetic probes mounted on the flux conserver wall are indicated with cross marks along the left wall. Probes are numbered according to their location along the wall. Location 1 is higher in the gun gap, while location 19 is at the bottom of the flux conserver.

- Figure 3. Initial plasma and spheromak formation sequence in SSPX. Magnetic measurements with the insertable probe (center-right) indicate currents flow along the expanding bubble. $B_{p}$ refers to the poloidal projection of vector field along the probe, and $B_{\theta}$ to the toroidal projection. Vectors show here the field orientation (not the magnitude) for a typical shot at this stage.

- Figure 4. (Color) Examples of plasma column kinks during formation. The SSPX shot number is shown in the upper-left corner of each frame, and the time after plasma breakdown in the lower-right corner. Hydrogen was used for shot 7978, while helium was used for shots 8176 and 8177. In all cases, the kink grows in a few microseconds, coinciding with the characteristic first large voltage spike like the one shown in Fig. 5. Visible light only. Images colored to illustrate light intensity levels.

- Figure 5. Gun current $\left(I_{g}\right)$ and voltage $\left(V_{g}\right)$ traces for formation-only shots. The images correspond to this shot, and represent an example of asymmetric plasma ejection. The bright feature at the center of the image at $55 \mu \mathrm{s}$ represents part of the $\sim 250 \mathrm{kA}$ current interacting with the probe' s boron nitride cover at that time.

- Figure 6. Toroidal and axial magnetic field averages for shots 15036 to 15051 with 
identical initial conditions. Time (with respect to breakdown) and $\lambda_{g}=\mu_{o} I_{g} j / \psi_{g}$, where $\psi_{g}$ is the gun flux, are indicated in the upper right-hand corner of every frame. The error bars indicate the shot-to-shot standard deviation, and do not take into account the systematic errors generated at the data acquisition system.

- Figure 7. (Color) (a) Gun current trace, (b) voltage trace, and (c) insertable probe $|B|=\sqrt{B_{r}^{2}+B_{\theta}^{2}+B_{z}^{2}}$ signals along the flux conserver radius. Each trace in (c) corresponds to a cluster location along the probe (color-coded in the inset scale). Magnetic signals closer to the machine axis have in general a higher $|B|$ amplitude. All signals from SSPX Shot 16209.

- Figure 8. (Color) Close-up of Fig. 7(c) showing a clear difference in the response between probes at the expected open flux surfaces, and probes at the closed ones.

- Figure 9. Helicity input rate from the plasma gun time-averaged over every pulse. Values shown are the mean and standard deviations for shots 16208 to 16210.

- Figure 10. Toroidal and axial magnetic field averages for shots 16208 to 16210 with identical initial conditions.

- Figure 11. Comparison of $|B|$ along the probe (labeled SIMP) with CORSICA reconstructions for Shot 16209 (Fig. 7). Time is shown in milliseconds in every frame. The data along the probe is joined by a solid line which gives the impression of having a serrated profile. However, this is caused by systematic errors (around $5 \%$ for all probe locations).

- Figure 12. Edge magnetic signals at different locations in the flux conserver (shown in Fig. 2). The measurements were taken: (solid line) shortly after the reconnection event of the third pulse of Fig. 7, and (dashed line) shortly before the next pulse. The error in the measurements is approximately $\pm 5 \%$ for all the channels.

- Figure 13. Helicity evolution for the fourth pulse of shots 16208 to 16210. Reconstruction averages from CORSICA are shown with the standard deviation for reconstruction result (which does not include an estimated $20 \%$ error with respect to the real quantities). The flux rope model prediction of helicity $K=2 n \Phi^{2}$ is shown at six different levels, where $\Phi$ is used at the start of the shot. 
- Figure 14. (Color) Cutaway view of the NIMROD simulation domain showing current density $\left(\times 10^{6} \mathrm{~A} / \mathrm{m}^{2}\right)$ during early spheromak formation. Times of each frame in microseconds. 

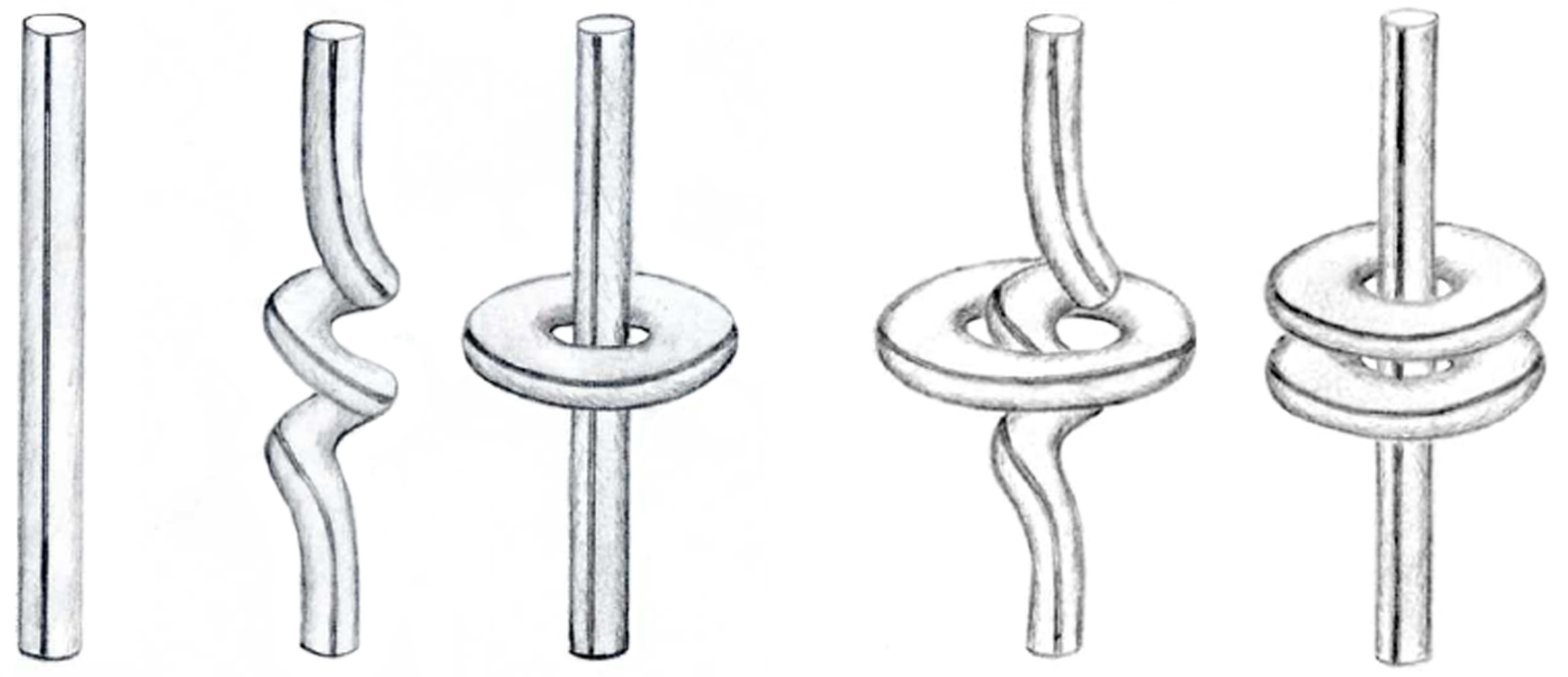

(a)

(b)

(c)

FIG. 1: 


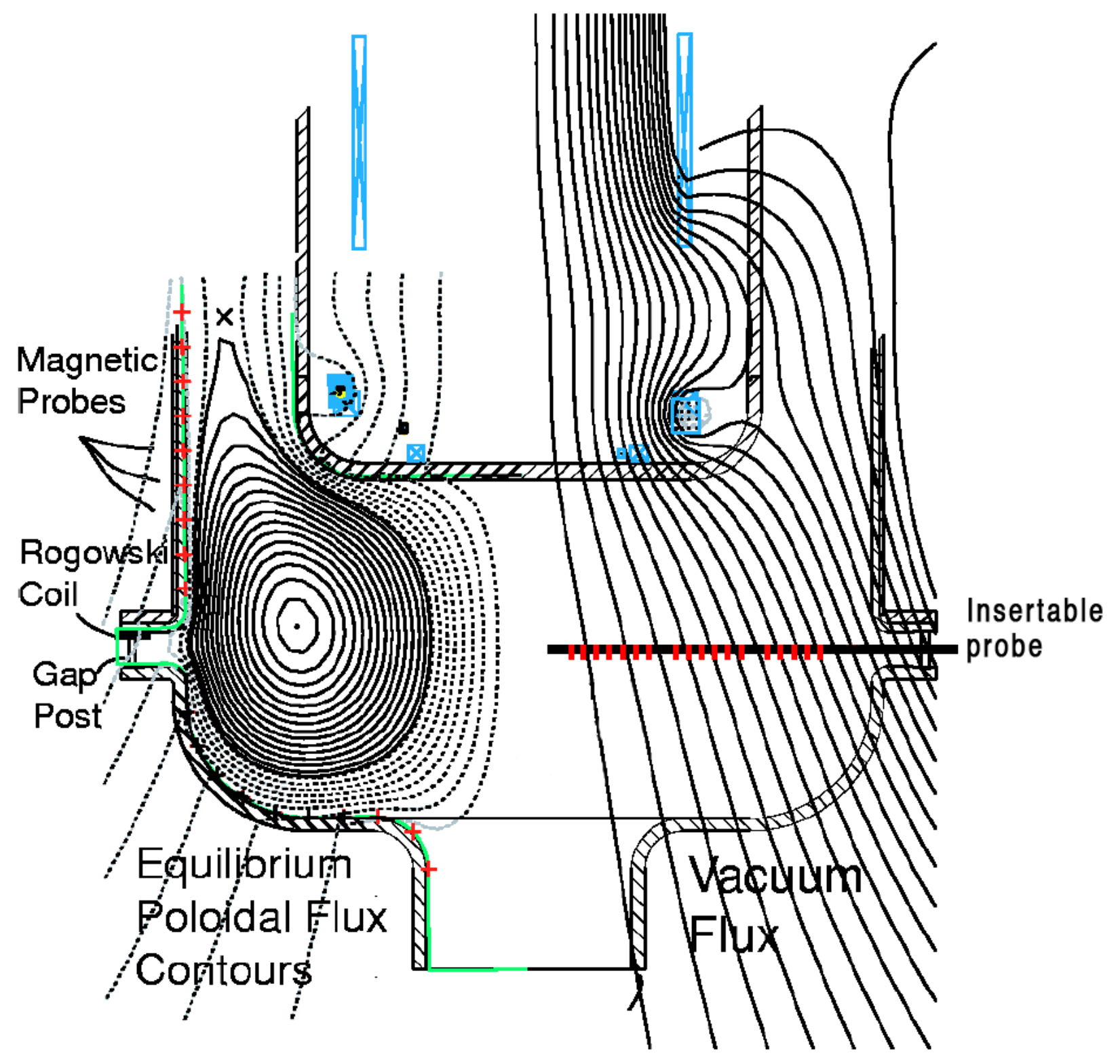

FIG. 2: 


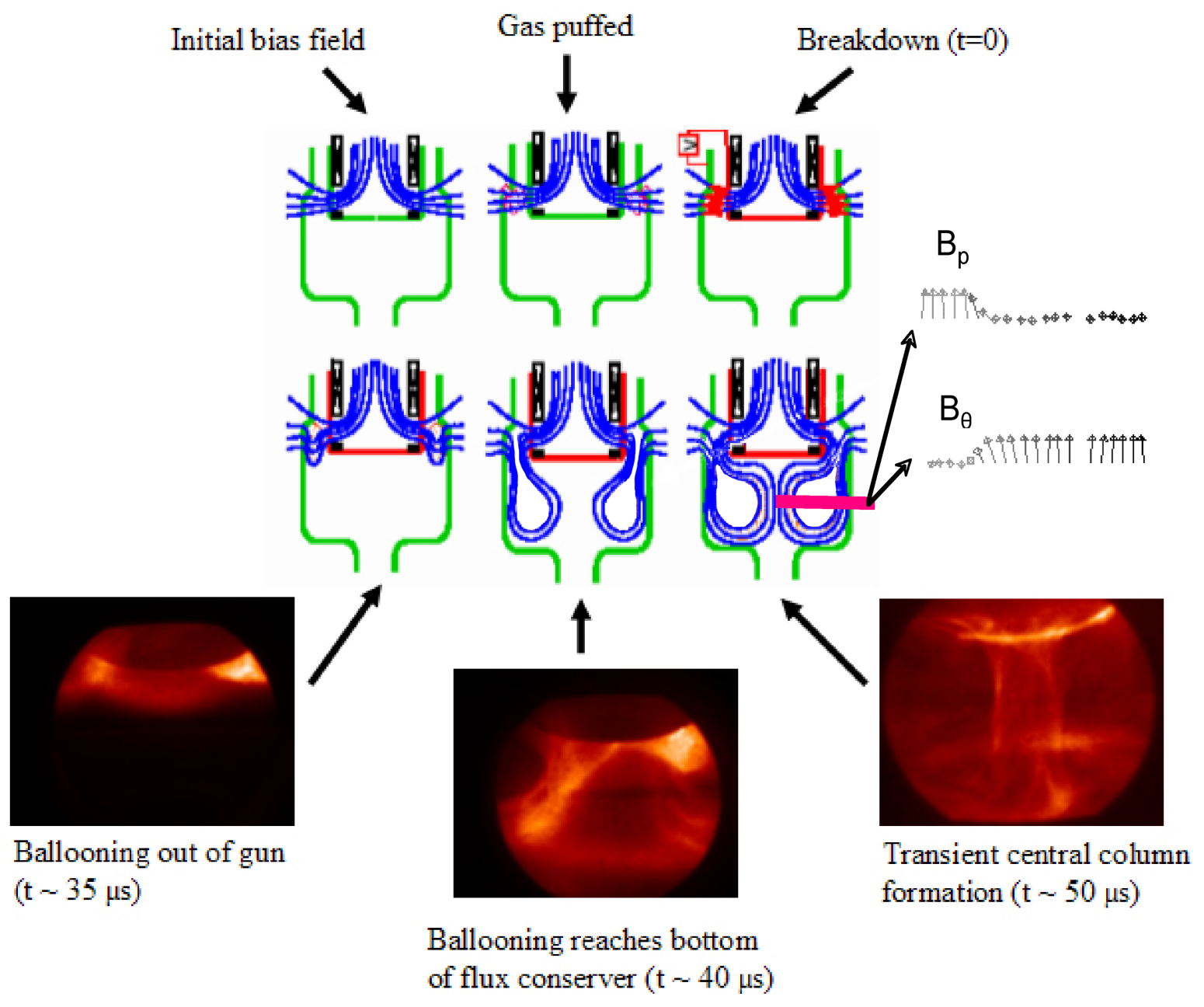

FIG. 3:
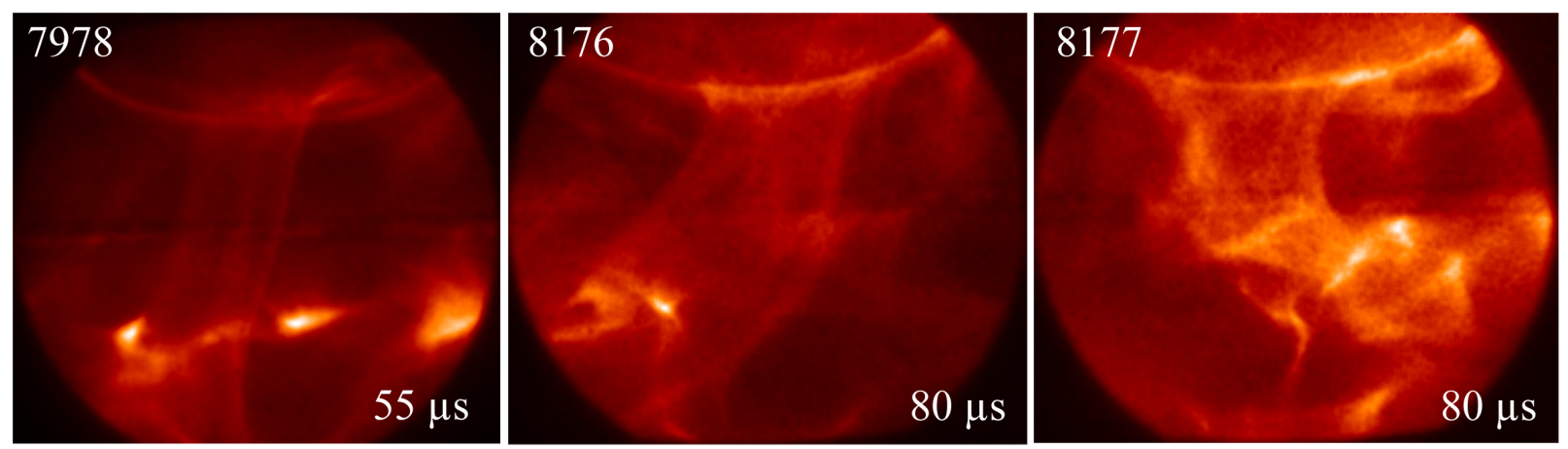

FIG. 4: 


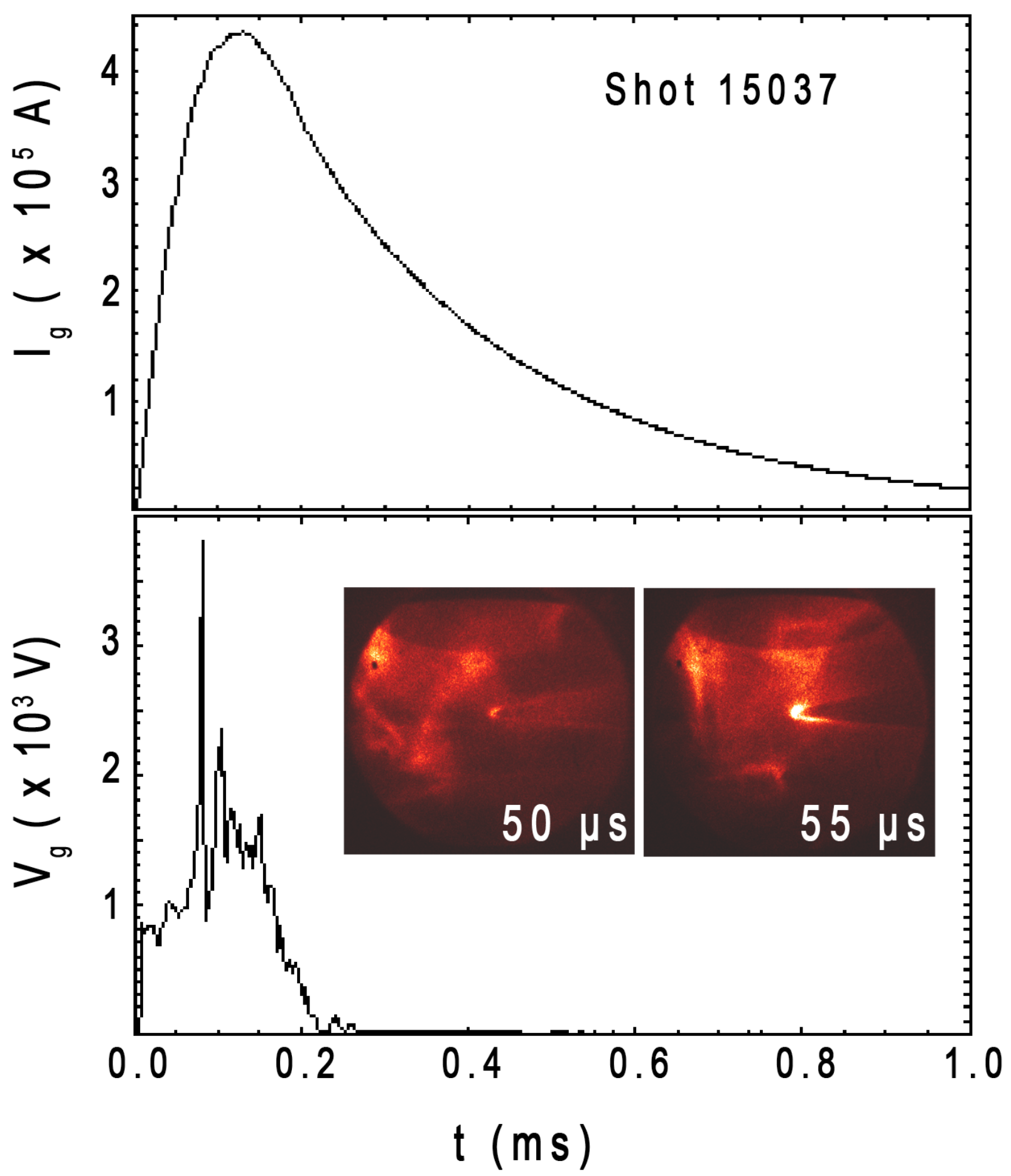

FIG. 5: 


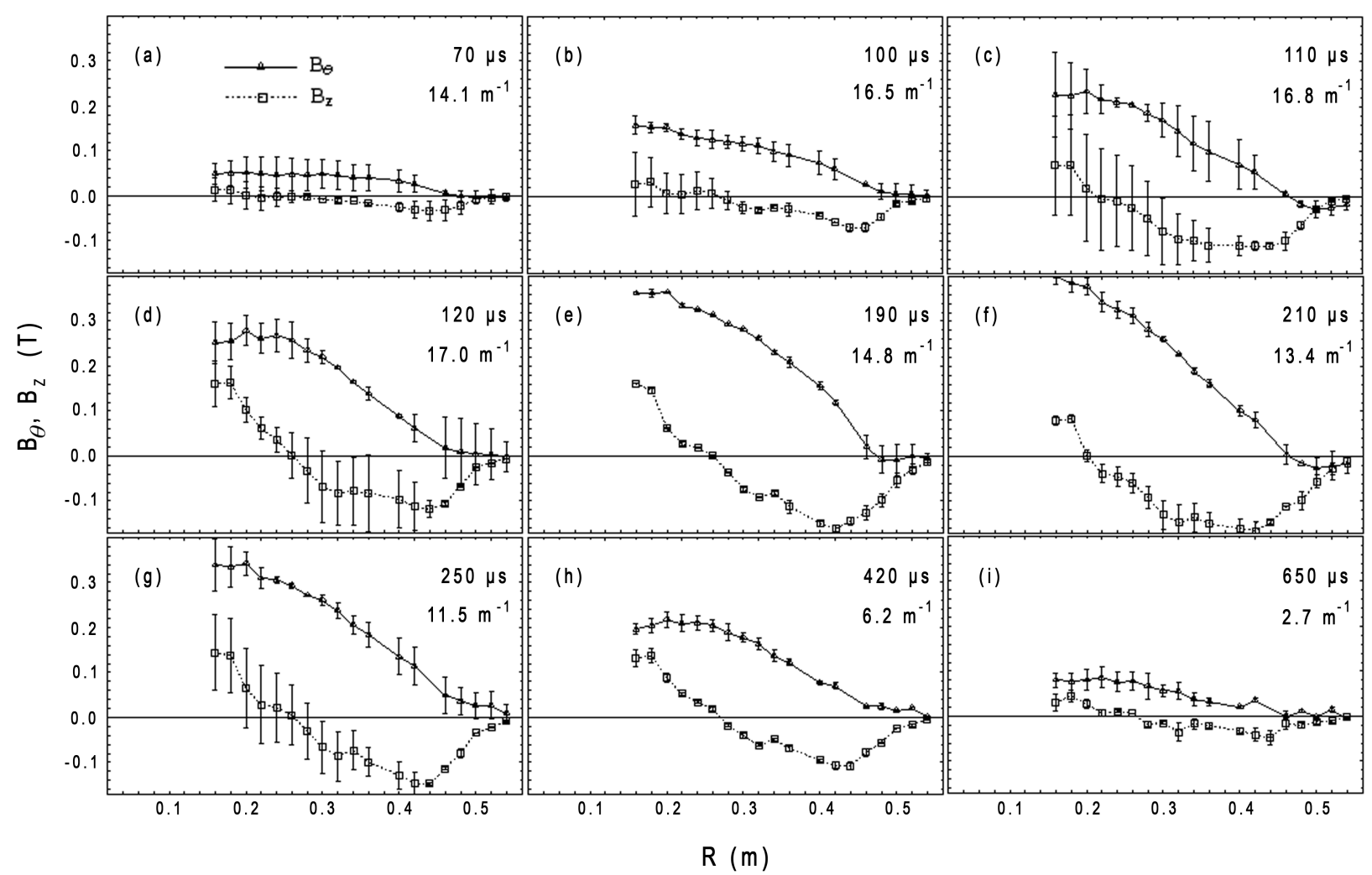

FIG. 6: 


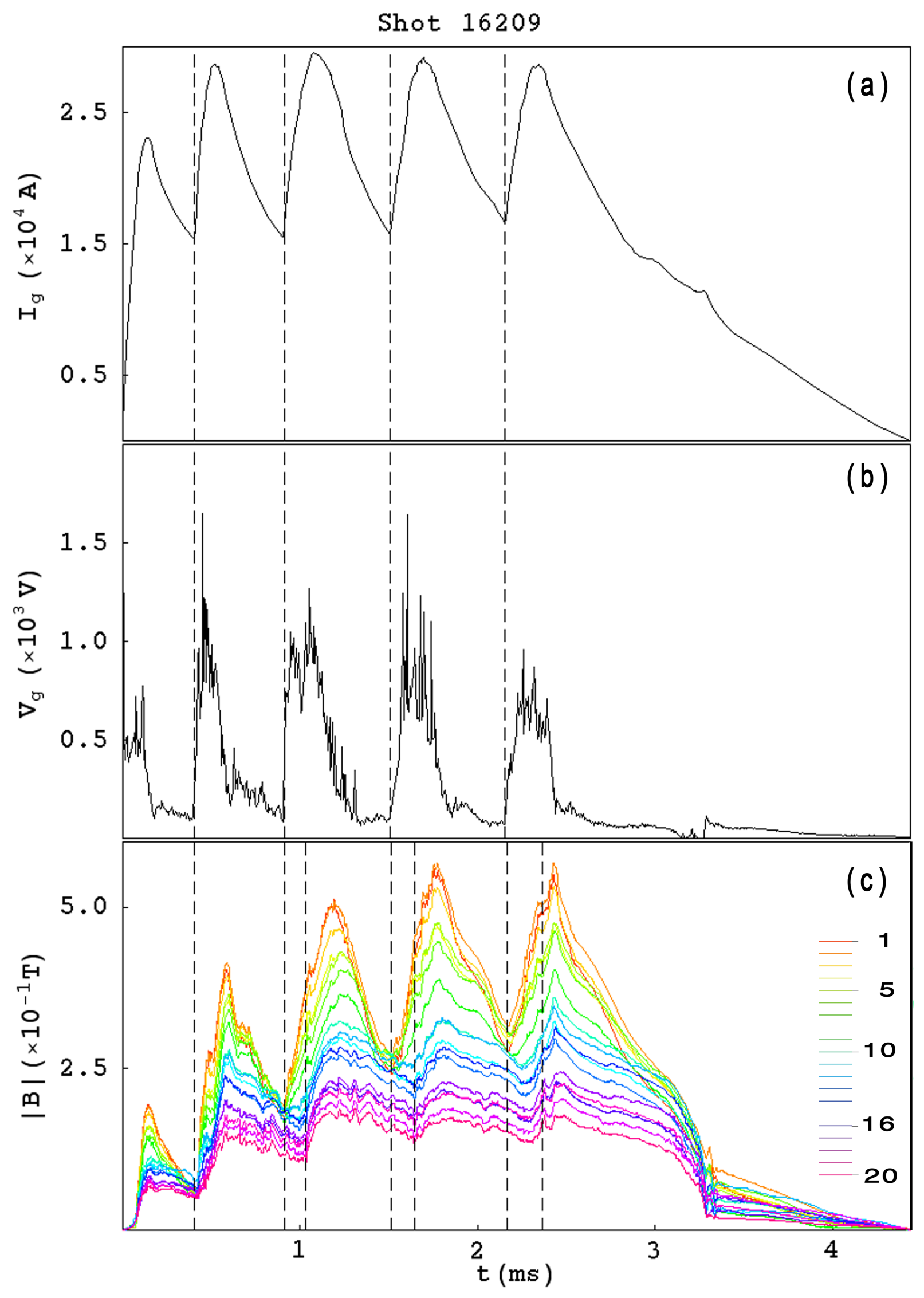

Fbg. 7: 


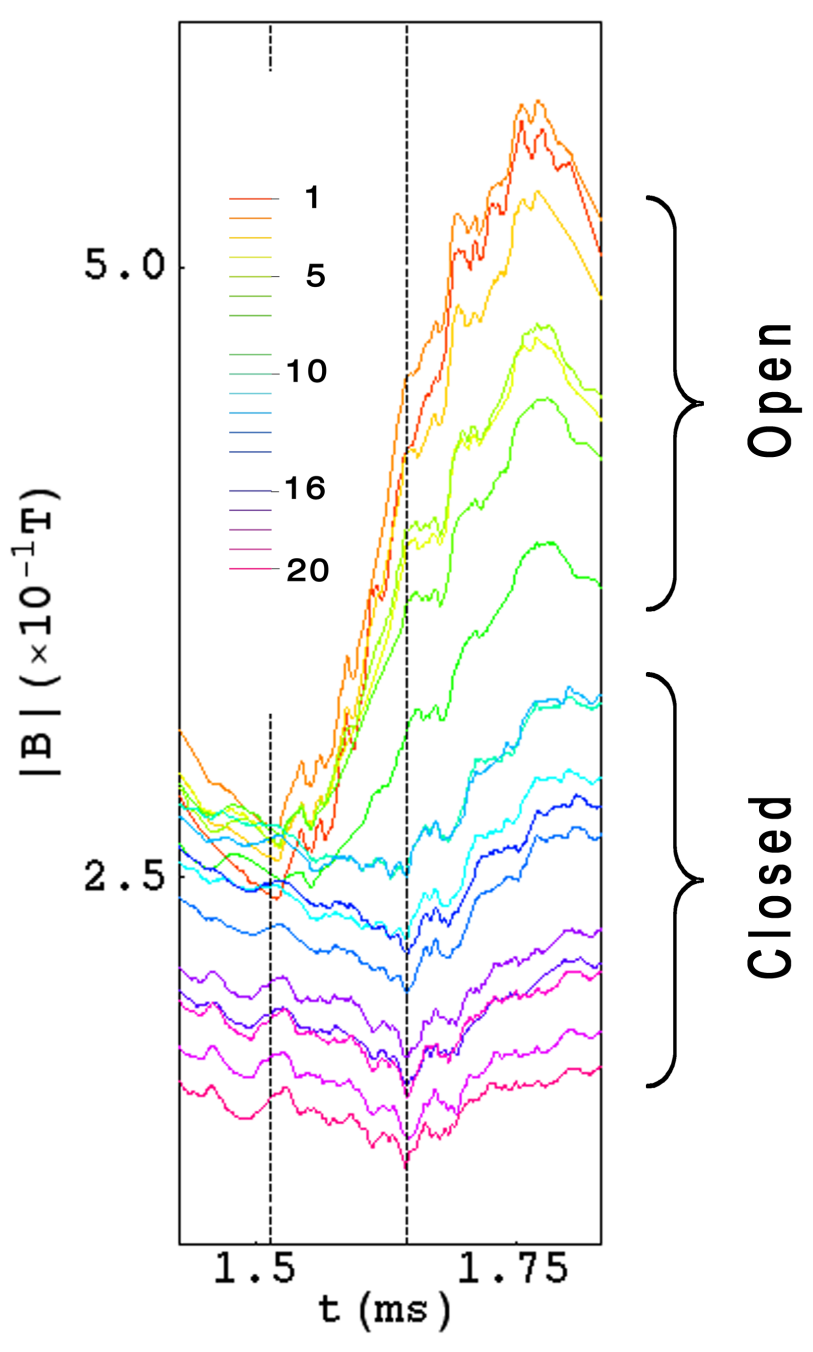

FIG. 8: 


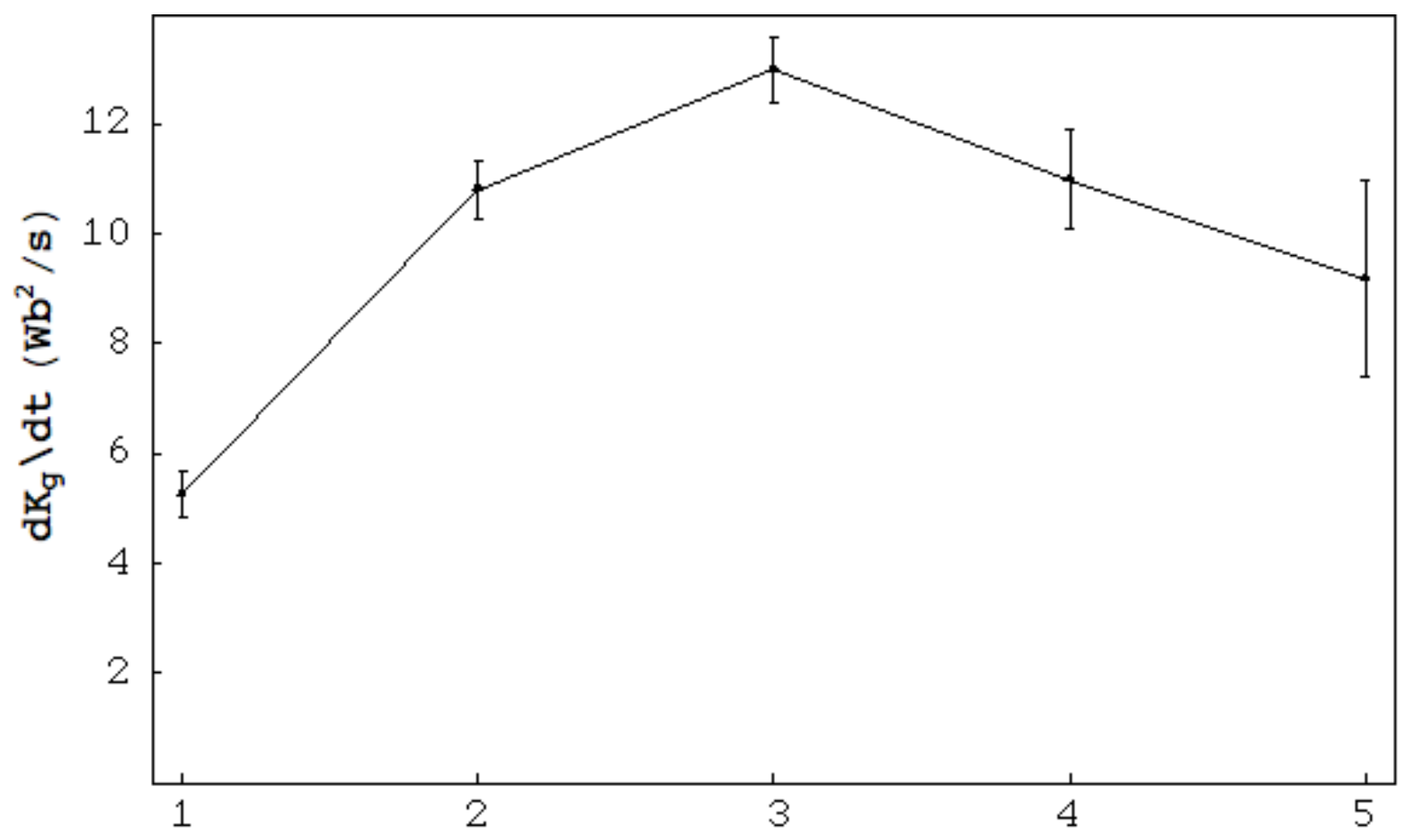

Gun Pulse Number

FIG. 9:

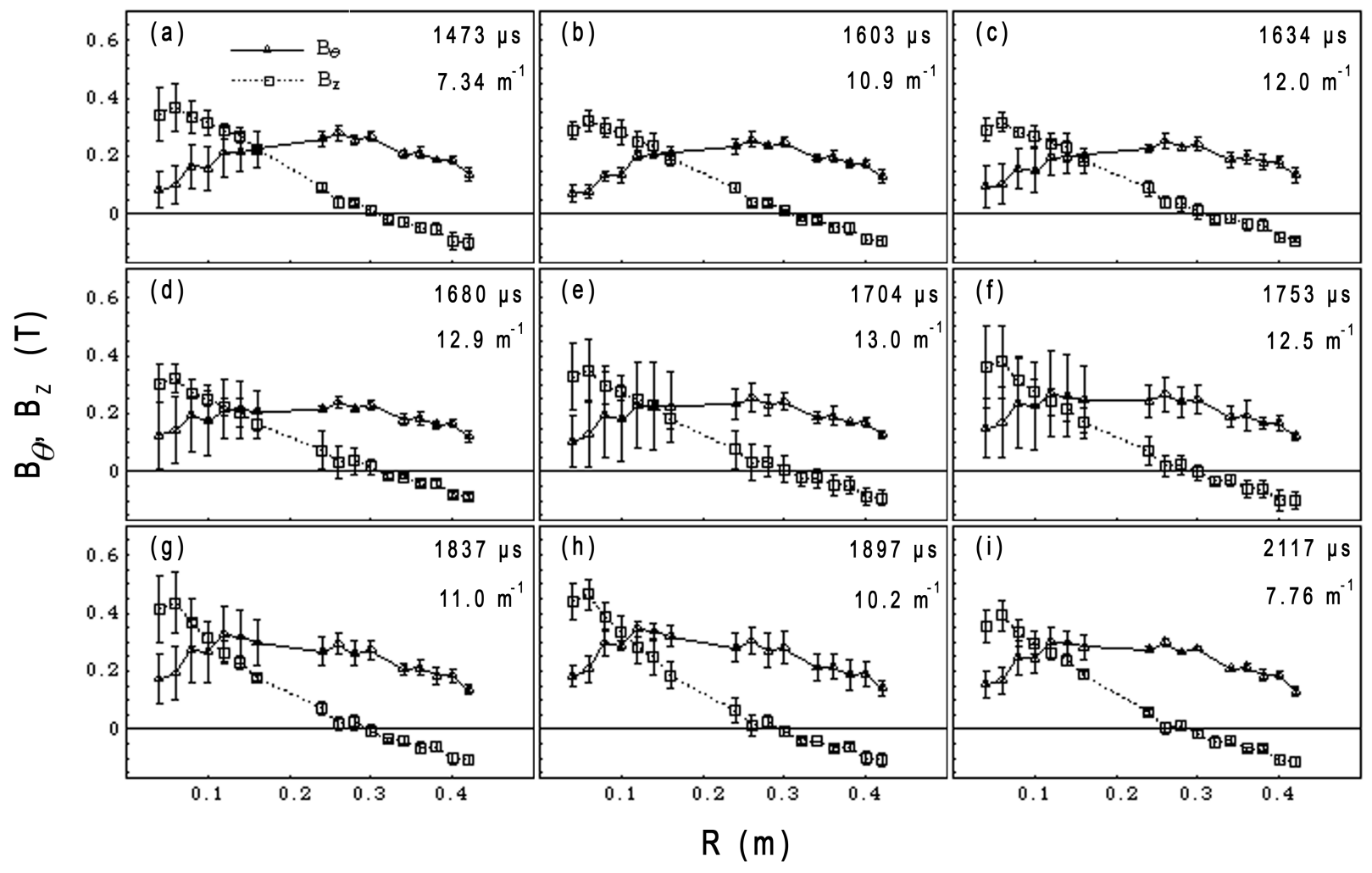

FIG. 10: 


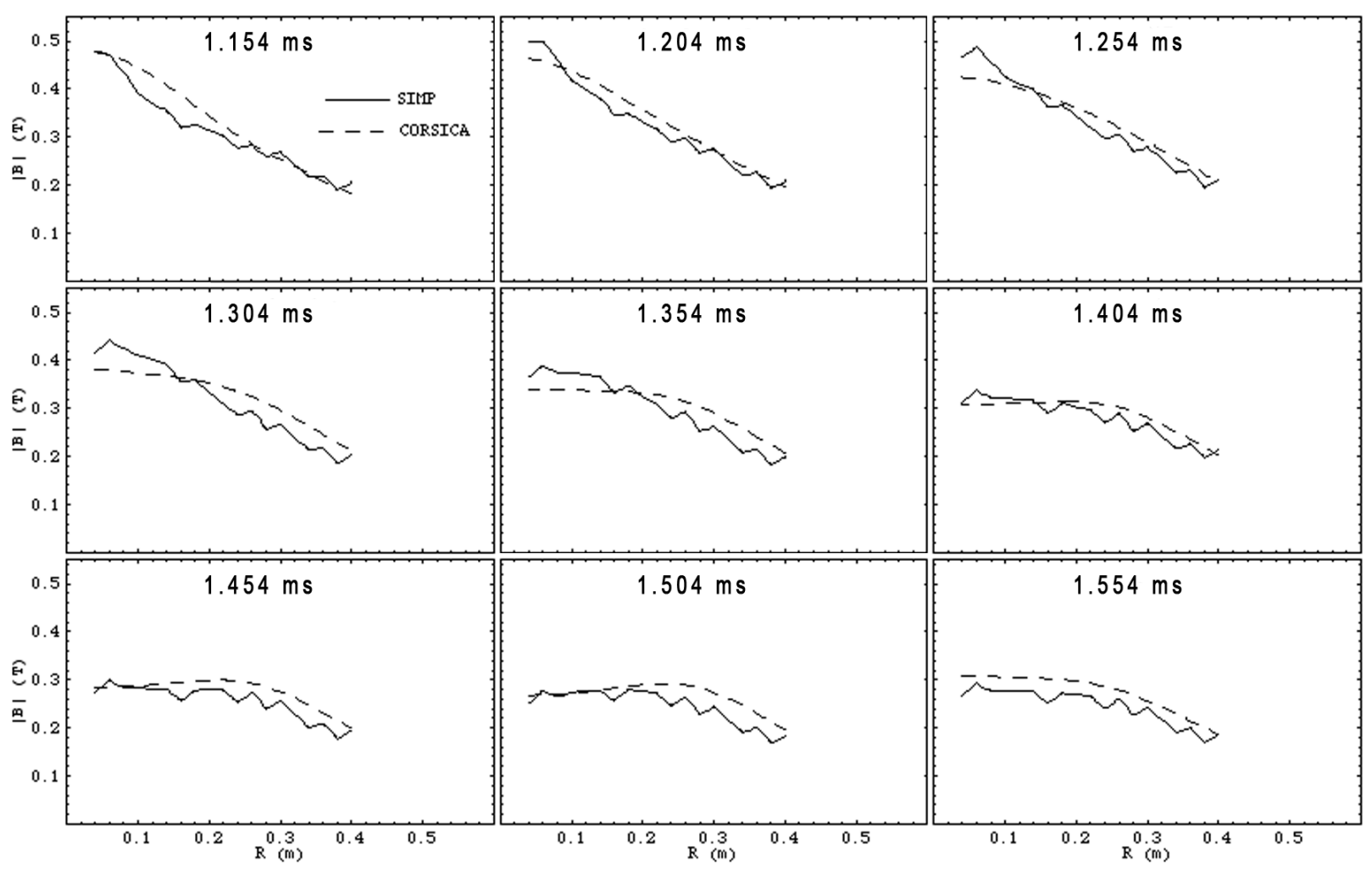

FIG. 11: 


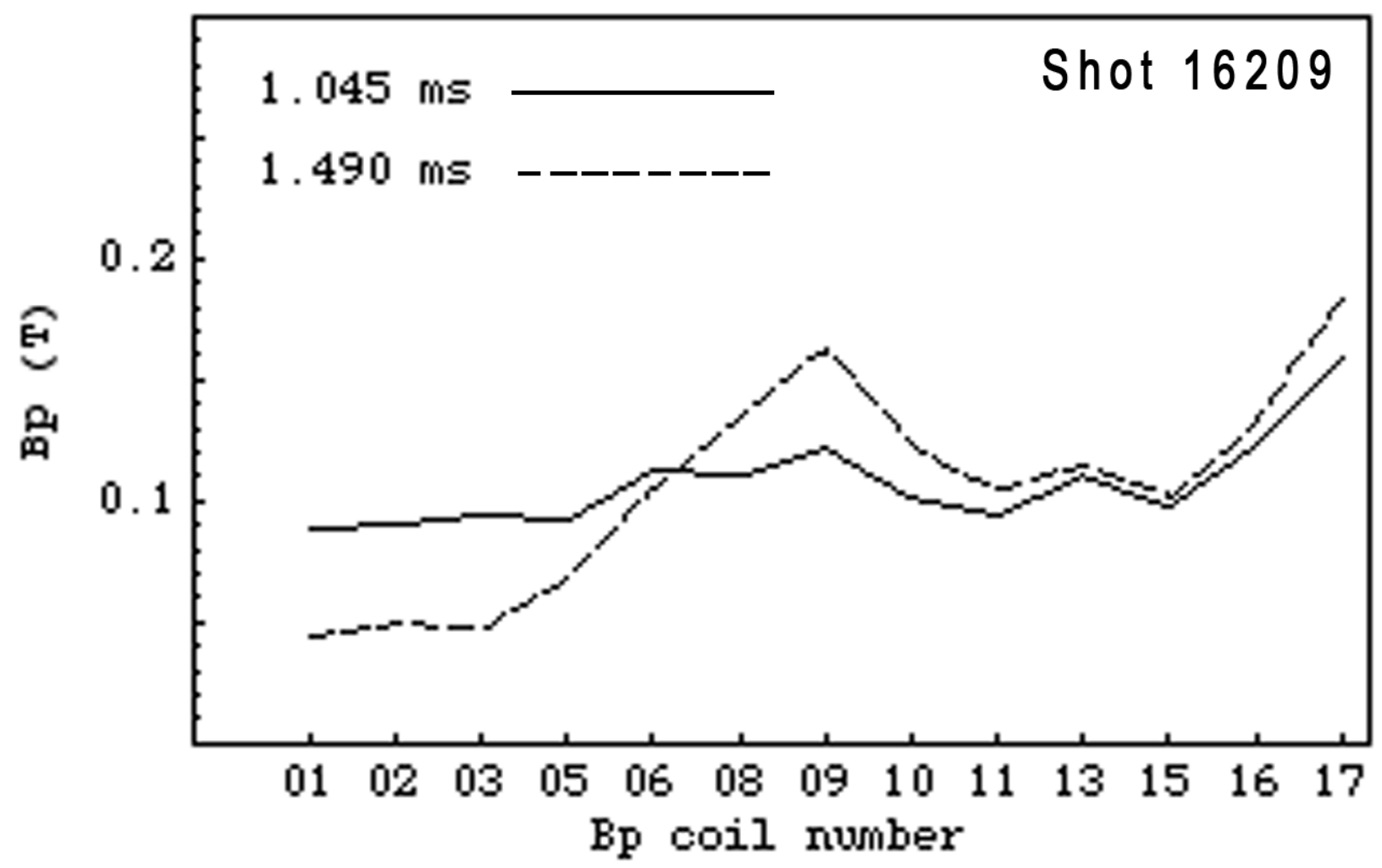

FIG. 12:

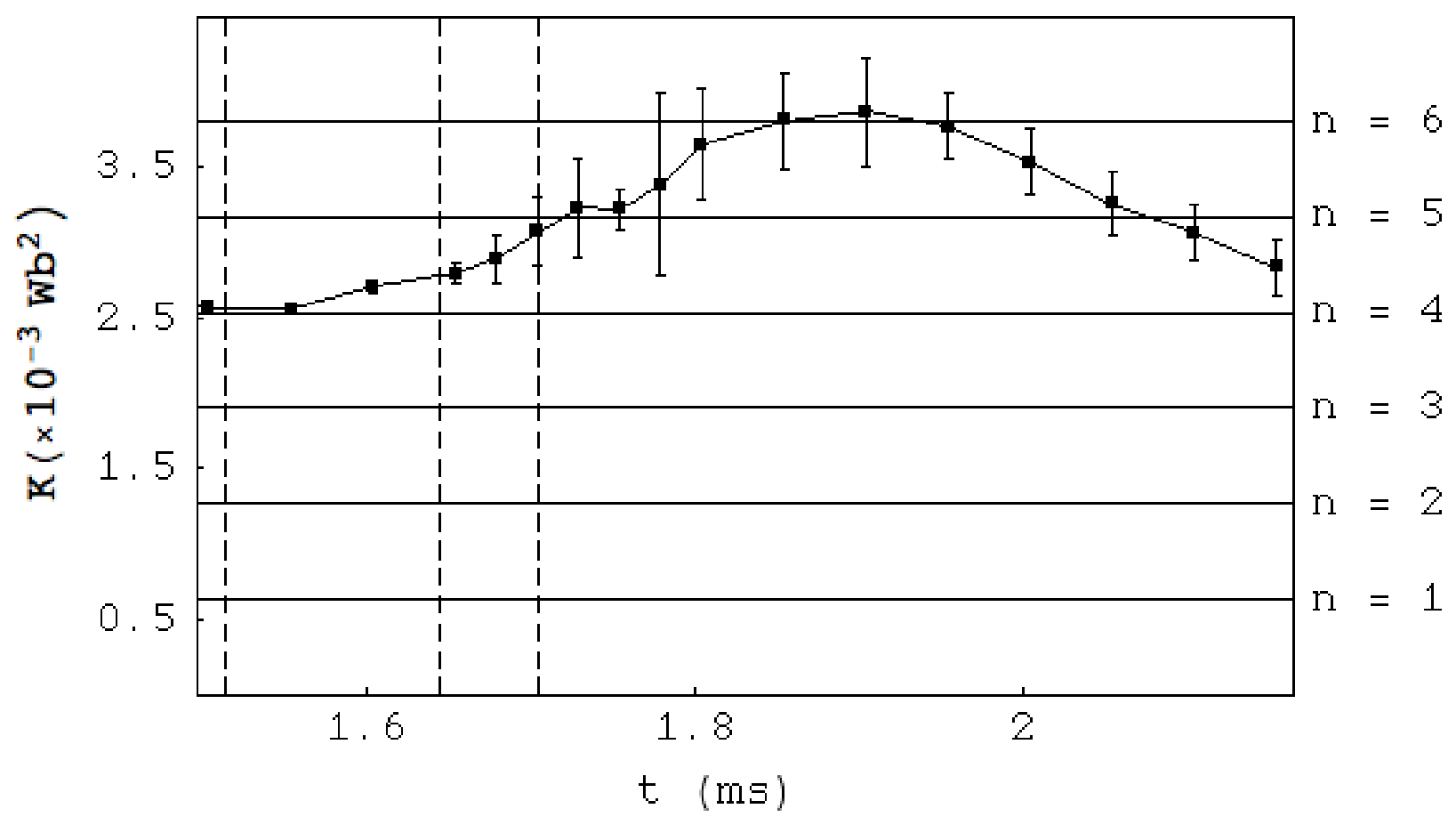

FIG. 13: 


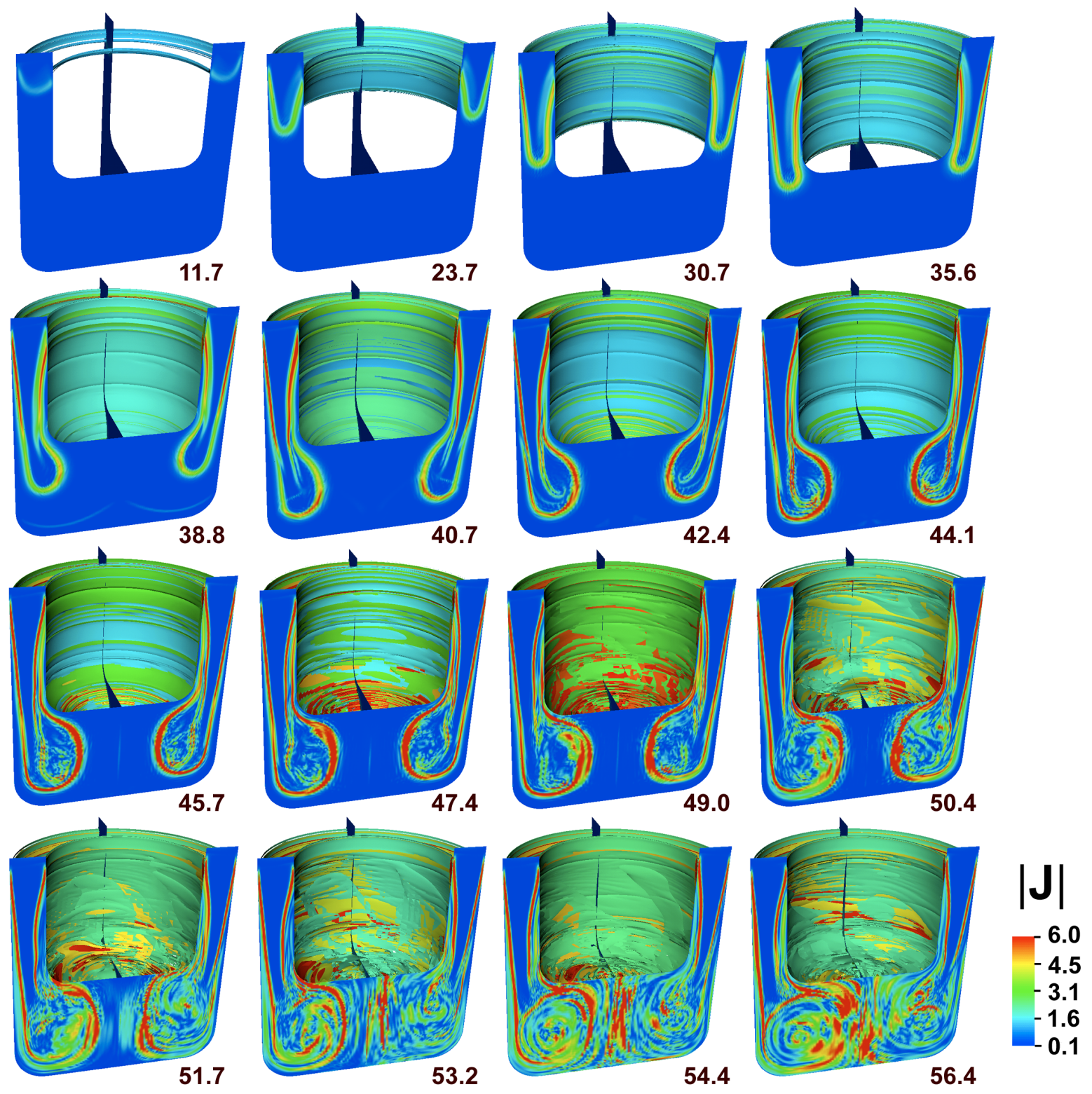

FIG. 14: 